\title{
A properly infinite Banach $*$-algebra with a non-zero, bounded trace
}

\author{
by \\ H. G. Dales (Leeds), Niels Jakob Laustsen (København) and \\ Charles J. Read (Leeds)
}

\begin{abstract}
A properly infinite $C^{*}$-algebra has no non-zero traces. We construct properly infinite Banach $*$-algebras with non-zero, bounded traces, and show that there are even such algebras which are fairly "close" to the class of $C^{*}$-algebras, in the sense that they can be hermitian or $*$-semisimple.
\end{abstract}

Introduction. Let $\mathscr{A}$ be a complex algebra. The commutator of two elements $a$ and $b$ in $\mathscr{A}$ is given by $[a, b]:=a b-b a$. A trace on $\mathscr{A}$ is a linear functional $\tau: \mathscr{A} \rightarrow \mathbb{C}$ satisfying

$$
\langle a b, \tau\rangle=\langle b a, \tau\rangle \quad(a, b \in \mathscr{A}) .
$$

Clearly, a trace maps each sum of commutators to 0, and, conversely, an element which is mapped to 0 by each trace is necessarily a sum of commutators. It follows that the space of traces on $\mathscr{A}$ encodes important information about the non-commutative structure of $\mathscr{A}$, and so it is of interest to study this space. In particular, it plays a significant role in the study of Lie derivations and other maps on Banach algebras; see [10] and [14], for example, for developments of this theory.

A unital algebra is properly infinite if it contains two idempotent elements which are orthogonal to each other and such that each is equivalent to the identity (see Definition 1.1, below, for details of the unexplained terminology). Fack has shown that each element in a unital, properly infinite $C^{*}$-algebra is the sum of five commutators (see [9, Theorem 2.1]), and so the zero functional is the only trace on a unital, properly infinite $C^{*}$-algebra. Recently, Pop has given a beautifully simple proof that two commutators in fact suffice (see [17]).

2000 Mathematics Subject Classification: Primary 46K05; Secondary 43A20, 46H20, $16 \mathrm{~N} 20$.

Key words and phrases: trace, properly infinite Banach algebra, Banach $*$-algebra, semigroup Banach algebra.

N. J. Laustsen is supported by the Danish Natural Science Research Council. 
In another direction, the second-named author has studied commutators of bounded linear operators on Banach spaces. Let $\mathfrak{X}$ be a Banach space, and denote by $\mathscr{B}(\mathfrak{X})$ the Banach algebra of all bounded linear operators on $\mathfrak{X}$. It is shown in [12, Proposition 3.7] that, in the case where $\mathfrak{X}$ is isomorphic to an infinite direct sum of copies of itself in a certain technical sense, each bounded linear operator on $\mathfrak{X}$ is the sum of two commutators, and so the zero functional is the only trace on $\mathscr{B}(\mathfrak{X})$ in this case. Prompted by this result, Villena raised the following question:

Let $\mathfrak{X}$ be a Banach space such that $\mathfrak{X}$ is isomorphic to $\mathfrak{X} \oplus \mathfrak{X}$. Is it true that the zero functional is the only trace on the Banach algebra $\mathscr{B}(\mathfrak{X})$ ?

This question is related to the existence of traces on properly infinite algebras because the Banach algebra $\mathscr{B}(\mathfrak{X})$ is properly infinite if and only if the Banach space $\mathfrak{X}$ contains a complemented subspace isomorphic to $\mathfrak{X} \oplus \mathfrak{X}$ (see [13, Proposition 1.9]). Taking account of this fact and of the above-mentioned results for $C^{*}$-algebras, the following question, raised in [12, Question 3.15], emerges as a natural generalization of Villena's question:

Let $\mathscr{A}$ be a unital, properly infinite algebra. Is it true that the zero functional is the only trace on $\mathscr{A}$ ?

In this paper we answer this question in the negative by constructing various unital, properly infinite Banach *-algebras, each with a non-zero, bounded trace. We first give an elementary example. However, this example is not semisimple, and we should like to have examples that are semisimple, and indeed "closer" to the class of $C^{*}$-algebras. For this we give a more complicated example that is $*$-semisimple, in the sense that it has a faithful *-representation on a Hilbert space.

1. Preliminaries. In this section we give the basic definitions, facts, and conventions that apply throughout this paper, and we provide further motivation for our work. Most of the results in this section are well known, but for completeness we have included some short and easy proofs.

Throughout, all algebras and vector spaces are assumed to be over the field $\mathbb{C}$ of complex numbers. Whenever convenient, we give definitions only for unital algebras; the reason for this is that all the concrete algebras to be studied in Sections 2-4 are indeed unital.

The monograph [7] is our standard reference on Banach algebras. Note in particular that we follow the convention of [7, Definition 3.1.1] by requiring that the involution on a Banach $*$-algebra be isometric, and that $\langle\cdot, \cdot\rangle$ denotes the pairing between a vector space and its dual space. 
1.1. Definition. Let $\mathscr{A}$ be an algebra. Two elements $a$ and $b$ in $\mathscr{A}$ are orthogonal to each other if $a b=0=b a$. An element $p$ in $\mathscr{A}$ is idempotent if $p^{2}=p$. Two idempotent elements $p$ and $q$ in $\mathscr{A}$ are algebraically equivalent, written $p \sim q$, if $p=a b$ and $q=b a$ for some elements $a$ and $b$ in $\mathscr{A}$.

Suppose that $\mathscr{A}$ has an identity $1_{\mathscr{A}}$. Then $\mathscr{A}$ is finite if $p=1_{\mathscr{A}}$ is the only idempotent element in $\mathscr{A}$ such that $p \sim 1_{\mathscr{A}}$. Otherwise, $\mathscr{A}$ is infinite. The algebra $\mathscr{A}$ is properly infinite if it contains two idempotent elements $p_{1}$ and $p_{2}$ which are orthogonal to each other and satisfy $p_{1} \sim 1_{\mathscr{A}} \sim p_{2}$. If $\mathscr{A}$ contains an idempotent element $p$ such that

$$
p \sim 1_{\mathscr{A}} \sim 1_{\mathscr{A}}-p,
$$

then we say that $\mathscr{A}$ is properly infinite in standard form.

We note that a unital, properly infinite algebra in standard form is indeed properly infinite. Moreover, it is easy to see that $\sim$ defines an equivalence relation on the set of idempotent elements, and that a unital algebra $\mathscr{A}$ is properly infinite if and only if there are elements $a_{1}, b_{1}, a_{2}$, and $b_{2}$ in $\mathscr{A}$ such that

$$
b_{j} a_{i}=\left\{\begin{array}{ll}
1_{\mathscr{A}} & \text { if } i=j \\
0 & \text { otherwise }
\end{array} \quad(i, j=1,2) .\right.
$$

1.2. ExAmples. (i) Each infinite von Neumann-algebra factor is properly infinite, and each properly infinite von Neumann algebra is in standard form. (E.g., see [11, Definition 6.3.1 and Lemma 6.3.3]; that our definition of properly infinite is equivalent to the one given in [11] follows from [7, Proposition 1.3.22(ii)], [11, Lemma 6.3.3], and the remarks following Definition 1.12 , below.)

(ii) The Cuntz $C^{*}$-algebra $\mathscr{O}_{n}$ introduced in [4] is properly infinite whenever $2 \leq n \leq \infty$, but only $\mathscr{O}_{2}$ is in standard form (see [5] or [6]).

(iii) Let $\mathfrak{X}$ be a Banach space. The Banach algebra $\mathscr{B}(\mathfrak{X})$ is properly infinite if and only if $\mathfrak{X}$ contains a complemented subspace isomorphic to $\mathfrak{X} \oplus \mathfrak{X}$ (see [13, Proposition 1.9]). In the case where $\mathfrak{X}$ is isomorphic to $\mathfrak{X} \oplus \mathfrak{X}, \mathscr{B}(\mathfrak{X})$ is in standard form.

1.3. Definition. A trace on an algebra $\mathscr{A}$ is a linear functional $\tau$ on $\mathscr{A}$ such that

$$
\langle a b, \tau\rangle=\langle b a, \tau\rangle \quad(a, b \in \mathscr{A}) .
$$

For a normed algebra $\mathscr{A}$, we write $\mathscr{T}(\mathscr{A})$ for the set of bounded traces on $\mathscr{A}$. This is the trace space of $\mathscr{A}$.

1.4. Traces on quotients. Let $\mathscr{I}$ be an ideal in an algebra $\mathscr{A}$. There is a canonical bijective correspondence between traces $\tau$ on $\mathscr{A}$ with $\mathscr{I} \subseteq \operatorname{ker} \tau$ 
and traces $\widehat{\tau}$ on $\mathscr{A} / \mathscr{I}$, illustrated by the commutative diagram

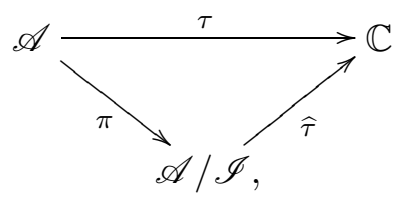

where $\pi$ denotes the quotient homomorphism. In the case where $\mathscr{I}$ is a closed ideal in a normed algebra $\mathscr{A}$, a trace $\tau$ on $\mathscr{A}$ with $\mathscr{I} \subseteq \operatorname{ker} \tau$ is bounded if and only if the corresponding trace $\widehat{\tau}$ on $\mathscr{A} / \mathscr{I}$ is bounded.

1.5. Definition. A linear functional $\lambda$ on an algebra $\mathscr{A}$ with an identity $1_{\mathscr{A}}$ is normalized if $\left\langle 1_{\mathscr{A}}, \lambda\right\rangle=1$.

1.6. Lemma. Let $\mathscr{A}$ be a unital, properly infinite algebra in standard form. Then the identity is the sum of two commutators, and there are no normalized traces on $\mathscr{A}$.

Proof. Take an idempotent element $p$ in $\mathscr{A}$ such that $p \sim 1_{\mathscr{A}} \sim 1_{\mathscr{A}}-p$, and take $a_{1}, b_{1}, a_{2}$, and $b_{2}$ in $\mathscr{A}$ such that $a_{1} b_{1}=p, a_{2} b_{2}=1_{\mathscr{A}}-p$, and $b_{1} a_{1}=1_{\mathscr{A}}=b_{2} a_{2}$. Then we have

$$
\left[b_{1}, a_{1}\right]+\left[b_{2}, a_{2}\right]=1_{\mathscr{A}}-p+1_{\mathscr{A}}-\left(1_{\mathscr{A}}-p\right)=1_{\mathscr{A}} .
$$

In particular, it follows that $\left\langle 1_{\mathscr{A}}, \tau\right\rangle=0$ for each trace $\tau$ on $\mathscr{A}$.

Given a unital, properly infinite algebra, we shall now describe a general method to produce a subalgebra which is unital and properly infinite in standard form. We are particularly interested in unital, properly infinite algebras in standard form because, in Villena's original question about the existence of non-zero traces on $\mathscr{B}(\mathfrak{X})$ in the case where $\mathfrak{X}$ is isomorphic to $\mathfrak{X} \oplus \mathfrak{X}$ (see p. 108), $\mathscr{B}(\mathfrak{X})$ is indeed properly infinite in standard form.

1.7. Definition. Let $p$ be an idempotent element in a unital algebra $\mathscr{A}$. The corner of $\mathscr{A}$ corresponding to $p$ is

$$
p \mathscr{A} p:=\{\text { pap } \mid a \in \mathscr{A}\} .
$$

The corner of a unital algebra $\mathscr{A}$ corresponding to an idempotent element $p$ is a subalgebra of $\mathscr{A}$ with identity $p$ (provided that $p \neq 0$ ). Suppose that $\mathscr{A}$ is a normed algebra. Then the subalgebra $p \mathscr{A} p$ is automatically closed in $\mathscr{A}$. However, the identity $p$ of $p \mathscr{A} p$ may have norm strictly greater than one, in which case we equip $p \mathscr{A} p$ with its left multiplier norm

$$
\||\|a \mid\|:=\sup \{\|a x\| \mid x \in p \mathscr{A} p,\|x\| \leq 1\} \quad(a \in p \mathscr{A} p) .
$$

This is an algebra norm equivalent to the original norm, and clearly $\|p\| \|=1$.

1.8. Proposition. Let $\mathscr{A}$ be a unital, properly infinite algebra. Then there is an idempotent element $p$ in $\mathscr{A}$ such that the corner $p \mathscr{A} p$ is properly infinite in standard form. 
Proof. Take idempotent elements $p_{1}$ and $p_{2}$ in $\mathscr{A}$ which are orthogonal to each other and satisfy $p_{1} \sim 1_{\mathscr{A}} \sim p_{2}$, and take elements $a_{1}, b_{1}, a_{2}$, and $b_{2}$ in $\mathscr{A}$ such that $a_{i} b_{i}=p_{i}$ and $b_{i} a_{i}=1_{\mathscr{A}}$ for $i=1,2$. Set $p:=1_{\mathscr{A}}-p_{1}$, $q:=p-p_{2}\left(=1_{\mathscr{A}}-p_{1}-p_{2}\right), c_{1}:=a_{2} p, d_{1}:=p b_{2}, c_{2}:=a_{2} a_{1} b_{2}+q$, and $d_{2}:=a_{2} b_{1} b_{2}+q$. Then $p$ and $q$ are idempotent, and $q, c_{1}, c_{2}, d_{1}$, and $d_{2}$ belong to $p \mathscr{A} p$. Direct calculations show that

$$
c_{1} d_{1}+c_{2} d_{2}=p \quad \text { and } \quad d_{j} c_{i}=\left\{\begin{array}{ll}
p & \text { if } i=j \\
0 & \text { otherwise }
\end{array} \quad(i, j=1,2),\right.
$$

and so the corner $p \mathscr{A} p$ is properly infinite in standard form.

For later reference, we record here how the spectrum of an element may change when passing to a corner. We write $\sigma_{\mathscr{A}}(a)$ for the spectrum of an element $a$ in an algebra $\mathscr{A}$, and we denote by $\nu_{\mathscr{A}}(a)$ its spectral radius. When no ambiguity arises, we shall omit the subscript " $\mathscr{A}$ ".

1.9. Lemma. Let $p$ be a non-zero, idempotent element in a unital algebra $\mathscr{A}$. For each element a in $p \mathscr{A} p$,

$$
\sigma_{p \mathscr{A} p}(a) \subseteq \sigma_{\mathscr{A}}(a) \subseteq \sigma_{p \mathscr{A} p}(a) \cup\{0\} .
$$

In particular, $\nu_{p \mathscr{A}}(a)=\nu_{\mathscr{A}}(a)$.

As explained in the introduction, it is our aim to find a unital, properly infinite Banach algebra $\mathscr{A}$ with a non-zero, bounded trace such that $\mathscr{A}$ is otherwise as "close" as possible to the class of $C^{*}$-algebras, where "close" means possessing standard properties. We shall now make this more explicit by defining the properties in which we are interested.

1.10. Definition. Let $\mathscr{A}$ be a unital $*$-algebra. Then:

(i) $\mathscr{A}$ is hermitian if $\sigma(a)$ is contained in $\mathbb{R}$ for each self-adjoint element $a$ in $\mathscr{A}$.

(ii) $\mathscr{A}$ is symmetric if $1_{\mathscr{A}}+a^{*} a$ is invertible for each $a$ in $\mathscr{A}$.

(iii) A linear functional $\lambda: \mathscr{A} \rightarrow \mathbb{C}$ is positive if $\left\langle a^{*} a, \lambda\right\rangle \geq 0$ for each $a$ in $\mathscr{A}$. A normalized, positive linear functional on $\mathscr{A}$ is called a state. A state which is also a trace is called a tracial state.

(iv) The $*$-radical of $\mathscr{A}$ is

$$
* \operatorname{rad} \mathscr{A}:=\left\{a \in \mathscr{A} \mid\left\langle a^{*} a, \lambda\right\rangle=0 \text { for each state } \lambda \text { on } \mathscr{A}\right\} .
$$

If $*$-rad $\mathscr{A}=\{0\}$, then $\mathscr{A}$ is $*$-semisimple.

A symmetric, unital $*$-algebra is hermitian. The converse is true for a unital Banach algebra with an involution; this is Shirali-Ford's theorem. The $*$-radical of a unital $*$-algebra $\mathscr{A}$ is a $*$-ideal in $\mathscr{A}$. In the case where $\mathscr{A}$ is a unital Banach $*$-algebra, the $*$-radical of $\mathscr{A}$ is automatically closed, it contains the radical of $\mathscr{A}$, and $\mathscr{A}$ is $*$-semisimple if and only if $\mathscr{A}$ has 
a faithful, unital *-representation on a Hilbert space (e.g., see [7, Proposition 1.10.22(iii), Theorem 3.1.10, Proposition 1.10.16(ii), p. 347, and Theorem 3.1.17]).

It is fundamental in the theory of $C^{*}$-algebras that a unital $C^{*}$-algebra is hermitian and $*$-semisimple (e.g., see [7, Proposition 3.2.3(v) and Corollary 3.2.13]), and that a unital $C^{*}$-algebra has a non-zero, bounded trace if and only if it has a tracial state. This leads us to consider the following questions.

1.11. Questions. Let $\mathscr{A}$ be a unital, properly infinite Banach $*$-algebra with a non-zero, bounded trace. Is it possible that:

(i) $\mathscr{A}$ is hermitian?

(ii) $\mathscr{A}$ is $*$-semisimple?

(iii) $\mathscr{A}$ is both hermitian and $*$-semisimple?

(iv) $\mathscr{A}$ has a tracial state?

In Sections 2 and 3, we answer Questions 1.11(i) and (ii) in the affirmative. We have not been able to resolve Questions 1.11(iii) and (iv); we can only show that none of the unital, properly infinite Banach $*$-algebras considered in this paper provides a positive answer to any of these questions. In the case of Question 1.11(iv), this relies on the following, formally stronger, version of Definition 1.1 for $*$-algebras.

1.12. Definition. Let $\mathscr{A}$ be a $*$-algebra. A self-adjoint, idempotent element in $\mathscr{A}$ is called a projection. Two projections $p$ and $q$ in $\mathscr{A}$ are Murray-von Neumann equivalent, written $p \approx q$, if $p=a^{*} a$ and $q=a a^{*}$ for some element $a$ in $\mathscr{A}$.

Suppose that $\mathscr{A}$ is unital. Then the terms $*$-finite, $*$-infinite, $*$-properly infinite, and *-properly infinite in standard form are defined as in Definition 1.1, just with the term "idempotent element" replaced by "projection" and the relation $\sim$ replaced by $\approx$.

We note that $\approx$ is an equivalence relation on the set of projections. Murray-von Neumann equivalent projections are clearly algebraically equivalent, and so a unital, *-properly infinite $*$-algebra is properly infinite, and a unital, finite $*$-algebra is $*$-finite. None of these implications can be reversed in general, as we shall show in Section 4 .

However, in a symmetric, unital *-algebra, each idempotent element is algebraically equivalent to a projection and, moreover, in a $C^{*}$-algebra, two projections are Murray-von Neumann equivalent if and only if they are algebraically equivalent (e.g., see [7, Propositions 1.10.21(i) and 3.2.10]). Along the same lines, one can show that a unital $C^{*}$-algebra is properly infinite (infinite, finite, respectively) if and only if it is $*$-properly infinite (*-infinite, $*$-finite, respectively). 
All our examples of unital, properly infinite Banach *-algebras are in fact $*$-properly infinite. This implies that they have no tracial states, as the following easy consequence of the Cauchy-Schwarz inequality (e.g., see [7, Proposition 1.10.13(iii)]) shows.

1.13. Lemma. Let $\lambda$ be a positive linear functional on a *-algebra $\mathscr{A}$ with an identity $1_{\mathscr{A}}$. Then $\lambda$ is non-zero if and only if $\left\langle 1_{\mathscr{A}}, \lambda\right\rangle$ is non-zero. In particular, $\mathscr{A}$ has a non-zero, positive trace if and only if $\mathscr{A}$ has a tracial state.

1.14. Corollary. There are no tracial states on a unital, *-properly infinite *-algebra.

Proof. Let $\tau$ be a positive trace on a unital, *-properly infinite $*$-algebra $\mathscr{A}$. Take projections $p_{1}$ and $p_{2}$ in $\mathscr{A}$ which are orthogonal to each other and satisfy $p_{1} \approx 1_{\mathscr{A}} \approx p_{2}$. Then $1_{\mathscr{A}}-p_{1}-p_{2}$ is a projection, and we have

$$
\left\langle p_{1}, \tau\right\rangle=\left\langle 1_{\mathscr{A}}, \tau\right\rangle=\left\langle p_{2}, \tau\right\rangle \geq 0 \quad \text { and } \quad\left\langle 1_{\mathscr{A}}-p_{1}-p_{2}, \tau\right\rangle \geq 0 .
$$

This implies that $\left\langle 1_{\mathscr{A}}, \tau\right\rangle=0$, so that $\tau=0$ by Lemma 1.13.

We conclude this preliminary section with some basic facts about corners in *-algebras.

1.15. Proposition. Let $\mathscr{A}$ be a unital $*$-algebra.

(i) For each projection $p$ in $\mathscr{A}$, the corner $p \mathscr{A} p$ is a sub-*-algebra of $\mathscr{A}$.

(ii) Suppose that $\mathscr{A}$ is $*$-properly infinite. Then $\mathscr{A}$ contains a projection $p$ such that the corner $p \mathscr{A} p$ is $*$-properly infinite in standard form.

(iii) Suppose that $\mathscr{A}$ is hermitian. Then, for each non-zero projection $p$ in $\mathscr{A}$, the corner $p \mathscr{A} p$ is hermitian.

(iv) Suppose that $\mathscr{A}$ is *-semisimple. Then, for each non-zero projection $p$ in $\mathscr{A}$, the corner $\operatorname{AAp}$ is $*$-semisimple.

Proof. Clause (i) is clear, clause (ii) follows from an obvious modification of the proof of Proposition 1.8, and clause (iii) is immediate from Lemma 1.9.

Clause (iv) is a consequence of [15, Theorem 9.7.17(a)], or it can be proved easily as follows. Let $p$ be a non-zero projection in $\mathscr{A}$. For each non-zero element $a$ in $p \mathscr{A} p$, we can take a state $\kappa$ on $\mathscr{A}$ such that $\left\langle a^{*} a, \kappa\right\rangle$ is non-zero. Then $\lambda:=\kappa \mid p \mathscr{A} p$ is a non-zero, positive linear functional on $p \mathscr{A} p$. Lemma 1.13 implies that $\langle p, \lambda\rangle$ is non-zero, and so $\mu:=\lambda /\langle p, \lambda\rangle$ is a state on $p \mathscr{A} p$ such that $\left\langle a^{*} a, \mu\right\rangle$ is non-zero. Hence $a$ is not in $*-\operatorname{rad}(p \mathscr{A} p)$.

2. An elementary and hermitian example. In this section we describe an elementary method to construct a unital, properly infinite Banach algebra with a non-zero, bounded trace. Moreover, we extend this method to encompass Banach *-algebras, and thus show that there exists a hermitian, unital, *-properly infinite Banach *-algebra with a non-zero, bounded trace. 
The corner stone is the following well known construction.

2.1. Construction. Let $\mathscr{A}$ be a Banach algebra, and let $\mathscr{X}$ be a Banach $\mathscr{A}$-bimodule. The Cartesian product $\mathscr{A} \oplus \mathscr{X}$ is a Banach algebra for the coordinatewise-defined vector-space operations, the multiplication

$$
(a, x)(b, y):=(a b, a \cdot y+x \cdot b) \quad(a, b \in \mathscr{A}, x, y \in \mathscr{X}),
$$

and the norm

$$
\|(a, x)\|:=\max \{\|a\|,\|x\|\} \quad(a \in \mathscr{A}, x \in \mathscr{X})
$$

(e.g., see [7, p. 239]).

Suppose that $\mathscr{A}$ has an identity $1_{\mathscr{A}}$, and that $\mathscr{X}$ is a unital Banach $\mathscr{A}$-bimodule in the sense that $1_{\mathscr{A}} \cdot x=x=x \cdot 1_{\mathscr{A}}$ for each $x$ in $\mathscr{X}$. Then $\left(1_{\mathscr{A}}, 0\right)$ is an identity in the Banach algebra $\mathscr{A} \oplus \mathscr{X}$.

2.2. Proposition. Let $\mathscr{A}$ be a unital Banach algebra, and let $\mathscr{X}$ be a unital Banach $\mathscr{A}$-bimodule. Then the Banach algebra $\mathscr{A} \oplus \mathscr{X}$ is properly infinite if and only if $\mathscr{A}$ is properly infinite. In this case, $\mathscr{A} \oplus \mathscr{X}$ is in standard form if and only if $\mathscr{A}$ is in standard form.

Proof. Suppose that the four elements $a_{1}, b_{1}, a_{2}$, and $b_{2}$ in $\mathscr{A}$ satisfy (1.1). Then we have

$$
\left(b_{j}, 0\right)\left(a_{i}, 0\right)=\left\{\begin{array}{ll}
\left(1_{\mathscr{A}}, 0\right) & \text { if } i=j \\
(0,0) & \text { otherwise }
\end{array} \quad(i, j=1,2),\right.
$$

and so $\mathscr{A} \oplus \mathscr{X}$ is properly infinite.

Conversely, suppose that $\left(a_{1}, x_{1}\right),\left(b_{1}, y_{1}\right),\left(a_{2}, x_{2}\right)$, and $\left(b_{2}, y_{2}\right)$ in $\mathscr{A} \oplus \mathscr{X}$ satisfy

$$
\left(b_{j}, y_{j}\right)\left(a_{i}, x_{i}\right)=\left\{\begin{array}{ll}
\left(1_{\mathscr{A}}, 0\right) & \text { if } i=j \\
(0,0) & \text { otherwise }
\end{array} \quad(i, j=1,2) .\right.
$$

Then certainly $a_{1}, b_{1}, a_{2}$, and $b_{2}$ satisfy (1.1), so that $\mathscr{A}$ is properly infinite.

The final clause is proved analogously.

2.3. Lemma. Let $\mathscr{A}$ be a Banach algebra, let $\mathscr{X}$ be a Banach $\mathscr{A}$-bimodule, and let $\lambda$ be a non-zero, bounded linear functional on $\mathscr{X}$. Then

$$
\tau_{\lambda}:(a, x) \mapsto\langle x, \lambda\rangle, \quad \mathscr{A} \oplus \mathscr{X} \rightarrow \mathbb{C},
$$

is a non-zero, bounded linear functional on $\mathscr{A} \oplus \mathscr{X}$, and $\tau_{\lambda}$ is a trace if and only if

$$
\langle a \cdot x, \lambda\rangle=\langle x \cdot a, \lambda\rangle \quad(a \in \mathscr{A}, x \in \mathscr{X}) .
$$

Proof. It is straightforward to see that $\tau_{\lambda}$ is non-zero, bounded, and linear. By the definitions, $\tau_{\lambda}$ is a trace if and only if

$$
\langle a \cdot x+y \cdot b, \lambda\rangle=\langle x \cdot a+b \cdot y, \lambda\rangle \quad(a, b \in \mathscr{A}, x, y \in \mathscr{X}) .
$$


This is certainly satisfied if (2.2) holds, and, conversely, taking $b=0$ and $y=0$ in (2.3) yields (2.2).

2.4. ExAmple. Let $\mathscr{A}$ be a unital Banach algebra. The projective tensor product $\mathscr{A} \widehat{\otimes} \mathscr{A}$ of two copies of $\mathscr{A}$ is a unital Banach $\mathscr{A}$-bimodule for maps which satisfy

$$
a \cdot(b \otimes c)=a b \otimes c \quad \text { and } \quad(b \otimes c) \cdot a=b \otimes c a \quad(a, b, c \in \mathscr{A})
$$

(e.g., see [7, Corollary 2.6.5]). Take a non-zero, bounded linear functional $\mu$ on $\mathscr{A}$. Then the map

$$
(a, b) \mapsto\langle b a, \mu\rangle, \quad \mathscr{A} \times \mathscr{A} \rightarrow \mathbb{C}
$$

is a non-zero, bounded bilinear form, and so there is a non-zero, bounded linear functional $\lambda: \mathscr{A} \widehat{\otimes} \mathscr{A} \rightarrow \mathbb{C}$ such that $\langle a \otimes b, \lambda\rangle=\langle b a, \mu\rangle$ for each $a$ and $b$ in $\mathscr{A}$. It follows that $\lambda$ satisfies condition (2.2) in Lemma 2.3 because

$$
\langle a \cdot(b \otimes c), \lambda\rangle=\langle c a b, \mu\rangle=\langle(b \otimes c) \cdot a, \lambda\rangle \quad(a, b, c \in \mathscr{A}),
$$

and so the functional $\tau_{\lambda}: \mathscr{A} \oplus(\mathscr{A} \widehat{\otimes} \mathscr{A}) \rightarrow \mathbb{C}$ defined by $(2.1)$ is a non-zero, bounded trace.

Combining Proposition 2.2 and Example 2.4 gives the following theorem, which answers [12, Question 3.15] in the negative.

2.5. THEOREM. Let $\mathscr{A}$ be a unital, properly infinite Banach algebra. Then $\mathscr{A} \oplus(\mathscr{A} \widehat{\otimes} \mathscr{A})$ is a unital, properly infinite Banach algebra which has a non-zero, bounded trace. Moreover, $\mathscr{A} \oplus(\mathscr{A} \widehat{\otimes} \mathscr{A})$ is in standard form if and only if $\mathscr{A}$ is in standard form.

2.6. REMARK. The above construction has some obvious limitations. Indeed, let $\mathscr{A}$ be a unital Banach algebra, and let $\mathscr{X}$ be a unital Banach $\mathscr{A}$-bimodule. Then:

(i) The Banach algebra $\mathscr{A} \oplus \mathscr{X}$ is never semisimple; the radical of $\mathscr{A} \oplus \mathscr{X}$ is given by

$$
\operatorname{rad}(\mathscr{A} \oplus \mathscr{X})=(\operatorname{rad} \mathscr{A}) \oplus \mathscr{X}
$$

(see [7, Theorem 1.8.14(ii)]). In Section 3 we shall use an entirely different approach to find an example of a semisimple, unital, properly infinite Banach algebra with a non-zero, bounded trace.

(ii) For each non-zero, bounded linear functional $\lambda$ on $\mathscr{X}$, we have

$$
\left\langle 1_{\mathscr{A} \oplus \mathscr{X}}, \tau_{\lambda}\right\rangle=\langle 0, \lambda\rangle=0 .
$$

It follows that $\tau_{\lambda}$ cannot be normalized, and Lemma 1.13 implies that this construction cannot be used to resolve Question 1.11(iv).

We shall now refine the above results to show that there is a hermitian, unital, *-properly infinite Banach *-algebra with a non-zero, bounded trace. We begin by extending Construction 2.1 to encompass Banach *-algebras. 
2.7. Construction. Let $\mathscr{A}$ be a Banach $*$-algebra, let $\mathscr{X}$ be a Banach $\mathscr{A}$-bimodule with an isometric linear involution, and suppose that the involutions on $\mathscr{A}$ and $\mathscr{X}$ are tied together through

$$
(a \cdot x)^{*}=x^{*} \cdot a^{*} \quad(a \in \mathscr{A}, x \in \mathscr{X}) .
$$

Then the Banach algebra $\mathscr{A} \oplus \mathscr{X}$ from Construction 2.1 is a Banach $*$-algebra for the involution

$$
(a, x)^{*}:=\left(a^{*}, x^{*}\right) \quad(a \in \mathscr{A}, x \in \mathscr{X}) .
$$

2.8. Example. Let $\mathscr{A}$ be a unital Banach $*$-algebra. The definition

$$
(a \otimes b)^{*}:=b^{*} \otimes a^{*} \quad(a, b \in \mathscr{A})
$$

induces an isometric linear involution on the Banach $\mathscr{A}$-bimodule $\mathscr{A} \widehat{\otimes} \mathscr{A}$ from Example 2.4, and (2.5) is satisfied in this case. Thus, $\mathscr{A} \oplus(\mathscr{A} \widehat{\otimes} \mathscr{A})$ is a unital Banach $*$-algebra for the involution given by (2.6).

2.9. Proposition. Let $\mathscr{A}$ be a unital Banach $*$-algebra, and let $\mathscr{X}$ be a unital Banach $\mathscr{A}$-bimodule with an isometric linear involution satisfying (2.5). Then:

(i) $\mathscr{A} \oplus \mathscr{X}$ is hermitian if and only if $\mathscr{A}$ is hermitian;

(ii) $\mathscr{A} \oplus \mathscr{X}$ is *-properly infinite if and only if $\mathscr{A}$ is $*$-properly infinite. In this case, $\mathscr{A} \oplus \mathscr{X}$ is in standard form if and only if $\mathscr{A}$ is in standard form.

Proof. By (2.4) (or a direct computation) we have

$$
\sigma_{\mathscr{A} \oplus \mathscr{X}}(a, x)=\sigma_{\mathscr{A}}(a) \quad(a \in \mathscr{A}, x \in \mathscr{X}) .
$$

Clause (i) is an immediate consequence of this. Clause (ii) is proved analogously to Proposition 2.2 .

Combining this with Examples 2.4 and 2.8 enables us to answer Question 1.11(i).

2.10. Corollary. Let $\mathscr{A}$ be a hermitian, unital, *-properly infinite Banach *-algebra. Then $\mathscr{A} \oplus(\mathscr{A} \widehat{\otimes} \mathscr{A})$ is a hermitian, unital, *-properly infinite Banach *-algebra with a non-zero, bounded trace. Moreover, the algebra $\mathscr{A} \oplus(\mathscr{A} \widehat{\otimes} \mathscr{A})$ is in standard form if and only if $\mathscr{A}$ is in standard form.

\section{A semisimple example}

3.1. Semigroup Banach algebras. Let $S$ be a semigroup. We use the monograph [3] as our standard reference on semigroups. The Banach space

$$
\ell_{1}(S):=\left\{f: S \rightarrow \mathbb{C}\left|\|f\|_{1}:=\sum_{s \in S}\right| f(s) \mid<\infty\right\},
$$


equipped with the pointwise-defined vector-space operations and the norm $\|\cdot\|_{1}$, is a Banach algebra for the convolution product $\star$ defined as follows. For each $s$ in $S$, let $\delta_{s}$ be the point mass at $s$, that is,

$$
\delta_{s}(t):=\left\{\begin{array}{ll}
1 & \text { if } s=t \\
0 & \text { otherwise }
\end{array} \quad(t \in S) .\right.
$$

Then each element $f$ of $\ell_{1}(S)$ can be uniquely expressed as an absolutely convergent sum $f=\sum_{s \in S} f(s) \delta_{s}$, and the convolution product is determined by the formula $\delta_{s} \star \delta_{t}:=\delta_{s t}$ for each $s$ and $t$ in $S$. We call $\ell_{1}(S)$ the semigroup Banach algebra of $S$. In the case where the semigroup $S$ has a neutral element $e$, the element $\delta_{e}$ is an identity in the algebra $\ell_{1}(S)$.

The dual space of $\ell_{1}(S)$ is canonically identified with the Banach space

$$
\ell_{\infty}(S):=\left\{\lambda: S \rightarrow \mathbb{C} \mid\|\lambda\|_{\infty}:=\sup \{|\lambda(s)| \mid s \in S\}<\infty\right\}
$$

via the pairing

$$
\langle f, \lambda\rangle:=\sum_{s \in S} f(s) \lambda(s) \quad\left(f \in \ell_{1}(S), \lambda \in \ell_{\infty}(S)\right) .
$$

In particular, we regard the trace space of $\ell_{1}(S)$ as a subspace of $\ell_{\infty}(S)$ :

$$
\mathscr{T}\left(\ell_{1}(S)\right)=\left\{\tau \in \ell_{\infty}(S) \mid \tau(s t)=\tau(t s)(s, t \in S)\right\} .
$$

3.2. Semigroups with a zero element. Let $S$ be a semigroup with at least two elements. An element $\theta$ in $S$ is a zero element if $s \theta=\theta=\theta s$ for each $s$ in $S$. (For technical reasons, it is convenient to exclude the trivial semigroup $\{0\}$ from the class of semigroups with zero element.) Clearly, a semigroup can have at most one zero element.

Let $\theta$ be a zero element in a semigroup $S$. We write $S$ - for $S \backslash\{\theta\}$. The closed ideal in $\ell_{1}(S)$ generated by $\delta_{\theta}$ is just $\mathbb{C} \delta_{\theta}$. It is often preferable to consider the quotient algebra $\ell_{1}^{\theta}(S):=\ell_{1}(S) / \mathbb{C} \delta_{\theta}$ instead of $\ell_{1}(S)$. As a Banach space, $\ell_{1}^{\theta}(S)$ is isometrically isomorphic to $\ell_{1}\left(S^{\mathbf{\square}}\right)$. The product, which we denote by $\widehat{\star}$, induced on $\ell_{1}\left(S^{\boldsymbol{\square}}\right)$ via this isometric isomorphism is determined by

$$
\delta_{s} \widehat{\star} \delta_{t}=\left\{\begin{array}{ll}
0 & \text { if } s t=\theta \\
\delta_{s t} & \text { otherwise }
\end{array} \quad\left(s, t \in S^{\mathbf{\square}}\right) .\right.
$$

It follows from Paragraph 1.4 that the trace space of $\ell_{1}\left(S^{\mathbf{\square}}\right)$ is given by

$$
\mathscr{T}\left(\ell_{1}\left(S^{\mathbf{\varpi}}\right)\right)=\left\{\tau|S \boldsymbol{\square}| \tau \in \mathscr{T}\left(\ell_{1}(S)\right) \text { with } \tau(\theta)=0\right\} \subseteq \ell_{\infty}\left(S^{\mathbf{\square}}\right) .
$$

3.3. Semigroups with an involution. An involution (or an involutorial anti-automorphism in the terminology of [3]) on a semigroup $S$ is a map $s \mapsto s^{*}, S \rightarrow S$, such that $\left(s^{*}\right)^{*}=s$ and $(s t)^{*}=t^{*} s^{*}$ for each $s$ and $t$ in $S$. An element $s$ in $S$ is self-adjoint if $s^{*}=s$.

Let $S$ be a semigroup with an involution. Then the formula

$$
f^{*}(s):=\overline{f\left(s^{*}\right)} \quad\left(f \in \ell_{1}(S), s \in S\right)
$$


defines an isometric involution on $\ell_{1}(S)$, so that $\ell_{1}(S)$ is a Banach $*$-algebra. Suppose that $S$ has a zero element $\theta$. Necessarily, $\theta$ is self-adjoint, and $\ell_{1}^{\theta}(S)$ inherits the isometric involution from $\ell_{1}(S)$. The ensuing isometric involution on $\ell_{1}\left(S^{\mathbf{\square}}\right)$ is still given by the formula (3.4), above, just with $f$ belonging to $\ell_{1}\left(S^{\mathbf{\square}}\right)$ and $s$ belonging to $S^{\boldsymbol{\square}}$.

3.4. Definition. Following Renault (see [18, p. 141ff.]), we denote by $S_{2}$ the second Cuntz semigroup, that is, $S_{2}$ is the semigroup with an involution $*$, neutral element $e$, zero element $\theta$, and generators $s_{1}, s_{2}, s_{1}^{*}$, and $s_{2}^{*}$ subject to the relations

$$
s_{i}^{*} s_{j}=\left\{\begin{array}{ll}
e & \text { if } i=j \\
\theta & \text { otherwise }
\end{array} \quad(i, j=1,2) .\right.
$$

3.5. Theorem. The unital Banach *-algebra $\ell_{1}\left(S_{2}\right)$ is *-properly infinite.

Proof. By (3.2) and (3.5), the elements $a_{1}:=\delta_{s_{1}}$ and $a_{2}:=\delta_{s_{2}}$ in $\ell_{1}\left(S_{2}^{\mathbf{\square}}\right)$ satisfy

$$
a_{j}^{*} \widehat{\star} a_{i}=\delta_{s_{j}^{*}} \widehat{\star} \delta_{s_{i}}=\left\{\begin{array}{ll}
\delta_{e} & \text { if } i=j \\
0 & \text { otherwise }
\end{array} \quad(i, j=1,2) .\right.
$$

In particular, Corollary 1.14 shows that there are no tracial states on $\ell_{1}\left(S_{2}\right)$. We shall next prove that, nevertheless, there are non-zero, bounded traces on $\ell_{1}\left(S_{2}\right)$. First we shall give a specific example of a normalized, bounded trace on $\ell_{1}\left(S_{2}^{\mathbf{M}}\right)$, and then we shall characterize all the bounded traces on $\ell_{1}\left(S_{2}^{\mathbb{\square}}\right)$ among the elements of $\ell_{\infty}\left(S_{2}^{\mathbf{\Xi}}\right)$.

3.6. Definition. Set

$$
\mathbf{I}:=\{\emptyset\} \cup\left\{\left(i_{1}, \ldots, i_{n}\right) \mid n \in \mathbb{N}, i_{1}, \ldots, i_{n} \in\{1,2\}\right\},
$$

and, for each $\mathbf{i}$ in $\mathbf{I}$, define an element $s_{\mathbf{i}}$ in $S_{2}$ as follows:

$$
s_{\mathbf{i}}:= \begin{cases}e & \text { for } \mathbf{i}=\emptyset, \\ s_{i_{1}} \ldots s_{i_{n}} & \text { for } \mathbf{i}=\left(i_{1}, \ldots, i_{n}\right) \in \mathbf{I} \backslash\{\emptyset\} .\end{cases}
$$

Moreover, for each $\mathbf{i}$ and $\mathbf{j}$ in $\mathbf{I}$, define $\mathbf{i j}$ in $\mathbf{I}$ by concatenation:

$$
\mathbf{i j}:= \begin{cases}\mathbf{i} & \text { for } \mathbf{j}=\emptyset, \\ \mathbf{j} & \text { for } \mathbf{i}=\emptyset \\ \left(i_{1}, \ldots, i_{m}, j_{1}, \ldots, j_{n}\right) & \text { for } \mathbf{i}=\left(i_{1}, \ldots, i_{m}\right) \text { and } \mathbf{j}=\left(j_{1}, \ldots, j_{n}\right) .\end{cases}
$$

Trivially, we have $s_{\mathbf{i}} s_{\mathbf{j}}=s_{\mathbf{i} \mathbf{j}}$ for each $\mathbf{i}$ and $\mathbf{j}$ in $\mathbf{I}$. More importantly, the following fundamental result, which is essentially due to Cuntz (see [4, Lemmas 1.2 and 1.3] or [16, formulae (1.4), (4.8), and (4.9)]), holds. 
3.7. Lemma. (i) For each $\mathbf{i}$ and $\mathbf{j}$ in $\mathbf{I}$,

$$
s_{\mathbf{i}}^{*} s_{\mathbf{j}}= \begin{cases}s_{\mathbf{k}}^{*} & \text { if } \mathbf{i}=\mathbf{j k} \text { for some } \mathbf{k} \in \mathbf{I} \\ s_{\mathbf{k}} & \text { if } \mathbf{j}=\mathbf{i k} \text { for some } \mathbf{k} \in \mathbf{I} \\ \theta & \text { otherwise. }\end{cases}
$$

(ii) For each $s$ in $S_{2}^{\bullet}$, there are unique $\mathbf{i}$ and $\mathbf{j}$ in $\mathbf{I}$ such that $s=s_{\mathbf{i}} s_{\mathbf{j}}^{*}$.

Lemma 3.7 gives a nuts-and-bolts description of the Banach-algebra structure of $\ell_{1}\left(S_{2}^{\square}\right)$. Indeed, clause (ii) implies that each $f$ in $\ell_{1}\left(S_{2}^{\mathbf{\square}}\right)$ has a unique representation as an absolutely convergent series of the form

$$
f=\sum_{\mathbf{i}, \mathbf{j} \in \mathbf{I}} \alpha_{\mathbf{i}, \mathbf{j}} \delta_{s_{\mathbf{i}} s_{\mathbf{j}}^{*}}
$$

where $\alpha_{\mathbf{i}, \mathbf{j}}=f\left(s_{\mathbf{i}} s_{\mathbf{j}}^{*}\right)$ for each $\mathbf{i}$ and $\mathbf{j}$ in $\mathbf{I}$, and $\|f\|_{1}=\sum\left|\alpha_{\mathbf{i}, \mathbf{j}}\right|$. Clause (i) then specifies how to multiply two elements represented as in (3.6).

We are now ready to construct a normalized, bounded trace on $\ell_{1}\left(S_{2}^{\boldsymbol{\varpi}}\right)$.

3.8. Example. Define $\tau$ in $\ell_{\infty}\left(S_{2}\right)$ by

$$
\tau(s)= \begin{cases}1 & \text { if } s=s_{\mathbf{i}} s_{\mathbf{i}}^{*} \text { for some } \mathbf{i} \in \mathbf{I} \quad\left(s \in S_{2}\right) . \\ 0 & \text { otherwise }\end{cases}
$$

Then $\tau$ is a normalized, bounded linear functional on $\ell_{1}\left(S_{2}\right)$. Lemma 3.7 implies that

$$
\tau(s t)= \begin{cases}1 & \text { if } s=s_{\mathbf{i j}} s_{\mathbf{k j}}^{*} \text { and } t=s_{\mathbf{k}} s_{\mathbf{i}}^{*} \text { for some } \mathbf{i}, \mathbf{j}, \mathbf{k} \in \mathbf{I} \\ & \text { or if } s=s_{\mathbf{i}} s_{\mathbf{j}}^{*} \text { and } t=s_{\mathbf{j k}} s_{\mathbf{i k}}^{*} \text { for some } \mathbf{i}, \mathbf{j}, \mathbf{k} \in \mathbf{I} \quad\left(s, t \in S_{2}\right) . \\ 0 & \text { otherwise }\end{cases}
$$

It follows that $\tau(s t)=\tau(t s)$ for each $s$ and $t$ in $S_{2}$, and so $\tau$ is a trace. Since $\tau(\theta)=0$, we conclude from (3.3) that $\tau \mid S_{2}^{\mathbf{Q}}$ is a normalized, bounded trace on $\ell_{1}\left(S_{2}^{\mathbf{a}}\right)$.

As mentioned above, our next aim is to determine all the bounded traces on $\ell_{1}\left(S_{2}^{\mathbf{\square}}\right)$.

3.9. Definition. For elements $s$ and $t$ in a semigroup $S$, we write $s \curlywedge t$ if there are elements $u$ and $v$ in $S$ such that $s=u v$ and $t=v u$.

Clearly $\curlywedge$ is a symmetric relation. In general, it need not be either reflexive or transitive. In the case of interest to us, that is, the second Cuntz semigroup $S_{2}$, it is indeed reflexive (because $S_{2}$ has a neutral element), but not transitive (for example, $s_{1} s_{2}^{*} \curlywedge \theta$ and $s_{2} s_{1}^{*} \curlywedge \theta$, but Lemma 3.7 implies that $\left.\neg\left(s_{1} s_{2}^{*} \curlywedge s_{2} s_{1}^{*}\right)\right)$.

Let $\widehat{\imath}$ be the smallest equivalence relation containing $\curlywedge$, and let

$$
[s]_{\widehat{\curlywedge}}:=\{t \in S \mid t \widehat{\curlywedge} s\}
$$

denote the $\widehat{\curlywedge}$-equivalence class of $s$ in $S$. The equivalence relation $\hat{\imath}$ has previously been studied in [2] (where it is denoted by $\approx$ ) in connection with 
investigations of the weak amenability of $\ell_{1}(S)$. The following proposition explains why it is relevant for our purposes.

3.10. Proposition. Let $S$ be a semigroup. An element $\tau$ in $\ell_{\infty}(S)$ is a trace on $\ell_{1}(S)$ if and only if $\tau(s)=\tau(t)$ whenever $s$ and $t$ in $S$ satisfy $s \widehat{\curlywedge} t$.

Proof. Suppose that $\tau$ is a trace. Define a relation $\curlywedge_{\tau}$ on $S$ by

$$
s \curlywedge_{\tau} t \Leftrightarrow \tau(s)=\tau(t) \quad(s, t \in S) .
$$

This is clearly an equivalence relation on $S$, and it contains $\curlywedge$ because $\tau$ is a trace. Consequently, $\curlywedge_{\tau}$ contains $\widehat{\curlywedge}$, that is, $\tau(s)=\tau(t)$ whenever $s$ and $t$ in $S$ satisfy $s \widehat{\curlywedge} t$.

Conversely, suppose that $\tau(s)=\tau(t)$ for each $s$ and $t$ in $S$ with $s \hat{\curlywedge} t$. Then, since $\widehat{\curlywedge}$ contains $\curlywedge$, we have $\tau(u v)=\tau(v u)$ for each $u$ and $v$ in $S$, and so $\tau$ is a trace by (3.1).

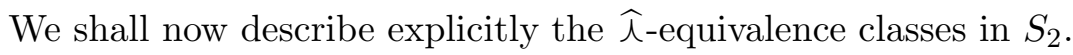

3.11. Lemma. (i) The $\widehat{\curlywedge}$-equivalence class of $\theta$ is given by

$$
\begin{aligned}
{[\theta]_{\widehat{\curlywedge}}=\{\theta\} \cup\left\{s_{\mathbf{i}} s_{\mathbf{j}}^{*} \mid \mathbf{i}=\left(i_{1}, \ldots, i_{m}\right), \mathbf{j}\right.} & =\left(j_{1}, \ldots, j_{n}\right) \in \mathbf{I} \backslash\{\emptyset\} \\
\text { with } i_{p} & \left.\neq j_{p} \text { for some } p \leq \min \{m, n\}\right\} .
\end{aligned}
$$

(ii) For each $\mathbf{i}$ in $\mathbf{I}$, the $\hat{\curlywedge}$-equivalence classes of $s_{\mathbf{i}}$ and $s_{\mathbf{i}}^{*}$ are given by

$$
\begin{aligned}
& {\left[s_{\mathbf{i}}\right]_{\widehat{\curlywedge}}=\left\{s_{\mathbf{h j k}} s_{\mathbf{h}}^{*} \mid \mathbf{h}, \mathbf{j}, \mathbf{k} \in \mathbf{I}, \mathbf{k j}=\mathbf{i}\right\},} \\
& {\left[s_{\mathbf{i}}^{*}\right]_{\widehat{\curlywedge}}=\left\{s_{\mathbf{h}} s_{\mathbf{h j k}}^{*} \mid \mathbf{h}, \mathbf{j}, \mathbf{k} \in \mathbf{I}, \mathbf{k j}=\mathbf{i}\right\} .}
\end{aligned}
$$

(iii) Each element of $S_{2}$ belongs to either $[\theta]_{\widehat{\curlywedge}},[e]_{\widehat{\curlywedge}},\left[s_{\mathbf{i}}\right]_{\widehat{\curlywedge}}$, or $\left[s_{\mathbf{i}}^{*}\right]_{\widehat{\curlywedge}}$ for some $\mathbf{i}$ in $\mathbf{I} \backslash\{\emptyset\}$.

Proof. Set

$$
\begin{aligned}
E(\theta)=\{\theta\} \cup\left\{s_{\mathbf{i}} s_{\mathbf{j}}^{*} \mid \mathbf{i}=\left(i_{1}, \ldots, i_{m}\right), \mathbf{j}\right. & =\left(j_{1}, \ldots, j_{n}\right) \in \mathbf{I} \backslash\{\emptyset\} \\
\text { with } i_{p} & \left.\neq j_{p} \text { for some } p \leq \min \{m, n\}\right\},
\end{aligned}
$$

and, for each $\mathbf{i}$ in $\mathbf{I}$, set

$$
\begin{aligned}
E\left(s_{\mathbf{i}}\right) & =\left\{s_{\mathbf{h j k}} s_{\mathbf{h}}^{*} \mid \mathbf{h}, \mathbf{j}, \mathbf{k} \in \mathbf{I}, \mathbf{k j}=\mathbf{i}\right\}, \\
E\left(s_{\mathbf{i}}^{*}\right) & =\left\{s_{\mathbf{h}} s_{\mathbf{h} \mathbf{j k}}^{*} \mid \mathbf{h}, \mathbf{j}, \mathbf{k} \in \mathbf{I}, \mathbf{k j}=\mathbf{i}\right\} .
\end{aligned}
$$

To prove that $E(x)$ is the $\widehat{\curlywedge}$-equivalence class of $x$ for each $x$ in the set $\left\{\theta, s_{\mathbf{i}}, s_{\mathbf{i}}^{*} \mid \mathbf{i} \in \mathbf{I}\right\}$, we introduce an equivalence relation $\tilde{\curlywedge}$ on $S_{2}$ whose equivalence class of $x$ is just $E(x)$, and then show that $\widetilde{\imath}$ is equal to $\widehat{\jmath}$.

Specifically, we observe that

$$
S_{2}=E(\theta) \cup \bigcup_{\mathbf{i} \in \mathbf{I}} E\left(s_{\mathbf{i}}\right) \cup \bigcup_{\mathbf{i} \in \mathbf{I}} E\left(s_{\mathbf{i}}^{*}\right),
$$


and that, for each $x$ and $y$ in $\left\{\theta, s_{\mathbf{i}}, s_{\mathbf{i}}^{*} \mid \mathbf{i} \in \mathbf{I}\right\}$, the two sets $E(x)$ and $E(y)$ are either equal or disjoint. This implies that the relation $\tilde{\imath}$ on $S_{2}$ defined by

$$
s \tilde{\lambda} t \Leftrightarrow s, t \in E(x) \text { for some } x \in\left\{\theta, s_{\mathbf{i}}, s_{\mathbf{i}}^{*} \mid \mathbf{i} \in \mathbf{I}\right\} \quad\left(s, t \in S_{2}\right)
$$

is an equivalence relation with equivalence classes

$$
[\theta]_{\tilde{\curlywedge}}=E(\theta), \quad\left[s_{\mathbf{i}}\right]_{\widetilde{\jmath}}=E\left(s_{\mathbf{i}}\right), \quad\left[s_{\mathbf{i}}^{*}\right]_{\widetilde{\jmath}}=E\left(s_{\mathbf{i}}^{*}\right) \quad(\mathbf{i} \in \mathbf{I}) .
$$

For each $x$ in $\left\{\theta, s_{\mathbf{i}}, s_{\mathbf{i}}^{*} \mid \mathbf{i} \in \mathbf{I}\right\}$ and each $s$ in $E(x)$, we have $s \curlywedge x$, and so $\hat{\imath}$ contains $\tilde{\curlywedge}$.

For the reverse inclusion, it suffices to prove that $\tilde{\imath}$ contains $\curlywedge$. Suppose that $s \curlywedge t$, where $s$ and $t$ belong to $S_{2}$, and take $u$ and $v$ in $S_{2}$ such that $s=u v$ and $t=v u$. We split the argument into four cases.

(1) $s=t=\theta$ : Then $s$ and $t$ both belong to $E(\theta)$.

(2) $s=\theta$ and $t \neq \theta$ : Since $v u=t \neq \theta$, necessarily $u \neq \theta$ and $v \neq \theta$, and, by Lemma 3.7, there are two subcases to consider.

(2a) $u=s_{\mathbf{h i}} s_{\mathbf{j}}^{*}$ and $v=s_{\mathbf{k}} s_{\mathbf{h}}^{*}$ for some $\mathbf{h}, \mathbf{i}, \mathbf{j}, \mathbf{k}$ in $\mathbf{I}$ : Since we have $\theta=s=u v=s_{\mathbf{h i}} s_{\mathbf{j}}^{*} s_{\mathbf{k}} s_{\mathbf{h}}^{*}$, Lemma 3.7(i) implies that $\mathbf{j}$ and $\mathbf{k}$ are non-empty, say $\mathbf{j}=\left(j_{1}, \ldots, j_{m}\right)$ and $\mathbf{k}=\left(k_{1}, \ldots, k_{n}\right)$, with $j_{p} \neq k_{p}$ for some $p \leq \min \{m, n\}$. Then $t=v u=s_{\mathbf{k i}} s_{\mathbf{j}}^{*}$ belongs to $E(\theta)$ because $\mathbf{k i}$ and $\mathbf{j}$ differ in the $p$ th coordinate.

(2b) $u=s_{\mathbf{h}} s_{\mathbf{i}}^{*}$ and $v=s_{\mathbf{j}} s_{\mathbf{h k}}^{*}$ for some $\mathbf{h}, \mathbf{i}, \mathbf{j}, \mathbf{k}$ in $\mathbf{I}$ : As above, we obtain $\mathbf{i}=\left(i_{1}, \ldots, i_{m}\right)$ and $\mathbf{j}=\left(j_{1}, \ldots, j_{n}\right)$ with $i_{p} \neq j_{p}$ for some $p \leq \min \{m, n\}$. It follows that $t=v u=s_{\mathbf{j}} s_{\mathbf{i k}}^{*}$ belongs to $E(\theta)$ because $\mathbf{j}$ and $\mathbf{i k}$ differ in the $p$ th coordinate.

(3) $s \neq \theta$ and $t=\theta$ : This is similar to case (2).

(4) $s \neq \theta$ and $t \neq \theta$ : By Lemma 3.7, there are four subcases to consider.

(4a) $u=s_{\mathbf{h}} s_{\mathbf{j i}}^{*}$ and $v=s_{\mathbf{j}} s_{\mathbf{h k}}^{*}$ for some $\mathbf{h}, \mathbf{i}, \mathbf{j}, \mathbf{k}$ in $\mathbf{I}$ : Then $s=s_{\mathbf{h}} s_{\mathbf{h k i}}^{*}$ and $t=s_{\mathbf{j}} s_{\mathbf{j i k}}^{*}$, which both belong to $E\left(s_{\mathbf{i k}}^{*}\right)$.

(4b) $u=s_{\mathbf{h i}} s_{\mathbf{k j}}^{*}$ and $v=s_{\mathbf{k}} s_{\mathbf{h}}^{*}$ for some $\mathbf{h}, \mathbf{i}, \mathbf{j}, \mathbf{k}$ in $\mathbf{I}$ : Then $s=s_{\mathbf{h i}} s_{\mathbf{h j}}^{*}$ and $t=s_{\mathbf{k i}} s_{\mathbf{k j}}^{*}$. By (3.7), we can take $x$ in $\left\{\theta, s_{\mathbf{g}}, s_{\mathbf{g}}^{*} \mid \mathbf{g} \in \mathbf{I}\right\}$ such that $s_{\mathbf{i}} s_{\mathbf{j}}^{*}$ belongs to $E(x)$. By the definition of $E(x)$, we have $s_{\mathbf{f}} E(x) s_{\mathbf{f}}^{*} \subseteq E(x)$ for each $\mathbf{f}$ in $\mathbf{I}$, and so $s$ and $t$ both belong to $E(x)$.

(4c) $u=s_{\mathbf{h}} s_{\mathbf{i}}^{*}$ and $v=s_{\mathbf{i j}} s_{\mathbf{h k}}^{*}$ for some $\mathbf{h}, \mathbf{i}, \mathbf{j}, \mathbf{k}$ in $\mathbf{I}$ : This is similar to subcase (4b).

(4d) $u=s_{\mathbf{h i}} s_{\mathbf{j}}^{*}$ and $v=s_{\mathbf{j k}} s_{\mathbf{h}}^{*}$ for some $\mathbf{h}, \mathbf{i}, \mathbf{j}, \mathbf{k}$ in $\mathbf{I}$ : Then $s=s_{\mathbf{h i k}} s_{\mathbf{h}}^{*}$ and $t=s_{\mathbf{j k i}} s_{\mathbf{j}}^{*}$, which both belong to $E\left(s_{\mathbf{i k}}\right)$.

In each of the above cases, we see that $s \tilde{\curlywedge} t$, as required.

We conclude that $\tilde{\curlywedge}=\widehat{\curlywedge}$, and clauses (i) and (ii) follow from (3.8).

Clause (iii) is a consequence of (i), (ii), and (3.7). 
Combining Proposition 3.10 and Lemma 3.11 yields the following characterization of the bounded traces on $\ell_{1}\left(S_{2}\right)$ among the elements of $\ell_{\infty}\left(S_{2}\right)$.

3.12. Proposition. An element $\tau$ in $\ell_{\infty}\left(S_{2}\right)$ is a trace on $\ell_{1}\left(S_{2}\right)$ if and only if it satisfies both of the following two conditions:

(i) $\tau(\theta)=\tau\left(s_{\mathbf{i}} s_{\mathbf{j}}^{*}\right)$ for each $\mathbf{i}=\left(i_{1}, \ldots, i_{m}\right)$ and $\mathbf{j}=\left(j_{1}, \ldots, j_{n}\right)$ in $\mathbf{I} \backslash\{\emptyset\}$ with $i_{p} \neq j_{p}$ for some $p \leq \min \{m, n\}$;

(ii) $\tau\left(s_{\mathbf{i}}\right)=\tau\left(s_{\mathbf{h j k}} s_{\mathbf{h}}^{*}\right)$ and $\tau\left(s_{\mathbf{i}}^{*}\right)=\tau\left(s_{\mathbf{h}} s_{\mathbf{h j k}}^{*}\right)$ for each $\mathbf{h}, \mathbf{i}, \mathbf{j}$, and $\mathbf{k}$ in $\mathbf{I}$ with $\mathbf{i}=\mathbf{k j}$.

By (3.3) and Proposition 3.12, we finally obtain the following characterization of the bounded traces on $\ell_{1}\left(S_{2}^{\mathbf{\bullet}}\right)$ among the elements of $\ell_{\infty}\left(S_{2}^{\mathbf{\Psi}}\right)$.

3.13. Corollary. An element $\tau$ in $\ell_{\infty}\left(S_{2}^{\mathbf{\square}}\right)$ is a trace on $\ell_{1}\left(S_{2}^{\mathbf{\square}}\right)$ if and only if it satisfies both of the following two conditions:

(i) $\tau\left(s_{\mathbf{i}} s_{\mathbf{j}}^{*}\right)=0$ for each $\mathbf{i}=\left(i_{1}, \ldots, i_{m}\right)$ and $\mathbf{j}=\left(j_{1}, \ldots, j_{n}\right)$ in $\mathbf{I} \backslash\{\emptyset\}$ with $i_{p} \neq j_{p}$ for some $p \leq \min \{m, n\}$;

(ii) $\tau\left(s_{\mathbf{i}}\right)=\tau\left(s_{\mathbf{h j k}} s_{\mathbf{h}}^{*}\right)$ and $\tau\left(s_{\mathbf{i}}^{*}\right)=\tau\left(s_{\mathbf{h}} s_{\mathbf{h j k}}^{*}\right)$ for each $\mathbf{h}, \mathbf{i}, \mathbf{j}$, and $\mathbf{k}$ in $\mathbf{I}$ with $\mathbf{i}=\mathbf{k j}$.

We shall next investigate which standard properties of a $C^{*}$-algebra $\ell_{1}\left(S_{2}\right)$ possesses. In particular, we shall show that it is $*$-semisimple.

3.14. Definition. An inverse semigroup is a semigroup $S$ such that, for each element $s$ in $S$, there is a unique element $t$ in $S$ with $s=s t s$ and $t=t s t$.

Let $S$ be an inverse semigroup. Then the map $s \mapsto t, S \rightarrow S$, where $t$ is the unique element in $S$ with $s=s t s$ and $t=t s t$, is an involution on $S$.

Barnes has proved in [1, Theorem 2.3] that, for each inverse semigroup $S$, the Banach $*$-algebra $\ell_{1}(S)$ has a faithful $*$-representation on a Hilbert space. Observe, however, that in the case where $S$ is an inverse semigroup with a zero element $\theta$, Barnes writes $\ell_{1}(S)$ for the Banach algebra that we denote by $\ell_{1}^{\theta}(S)$ (see [1, p. 365]), and so it follows from Barnes's work that $\ell_{1}\left(S^{\mathbf{\square}}\right)$ is *-semisimple. We note in passing that Wordingham has subsequently improved Barnes's result by showing that the left regular $*$-representation of $\ell_{1}(S)$ on $\ell_{2}(S)$ is faithful whenever $S$ is an inverse semigroup (see [20, Theorem 2] or [16, Theorem 2.1.1]). It is a well-known consequence of Lemma 3.7 that $S_{2}$ is an inverse semigroup (e.g., see [16, p. 3]), and so we obtain the following result.

3.15. Proposition. The unital Banach *-algebra $\ell_{1}\left(S_{2}\right)$ is $*$-semisimple. 
3.16. Remark. It is also possible to give an entirely elementary proof of Proposition 3.15. Indeed, let $\left(e_{m}: m \in \mathbb{N}\right)$ denote the canonical orthonormal basis for the Hilbert space $\ell_{2}(\mathbb{N})$, and set

$$
R_{i}\left(e_{m}\right):=e_{3(m-1)+i} \quad(m \in \mathbb{N}, i=1,2) .
$$

These definitions extend by linearity and continuity to give isometric operators $R_{1}$ and $R_{2}$ on $\ell_{2}(\mathbb{N})$. Set $\pi\left(\delta_{s_{i}}\right):=R_{i}$ for $i=1,2$. It is straightforward to see that this definition has a unique extension to a unital $*$-representation $\pi: \ell_{1}\left(S_{2}^{\mathbf{a}}\right) \rightarrow \mathscr{B}\left(\ell_{2}(\mathbb{N})\right)$, and that $\pi$ is faithful.

3.17. TheOREM. There is a *-semisimple, unital, *-properly infinite Banach *-algebra in standard form which has a non-zero, bounded trace. Specifically, the corner of $\ell_{1}\left(S_{2}^{\mathbf{\square}}\right)$ corresponding to the projection $\delta_{e}-\delta_{s_{1} s_{1}^{*}}$ has these properties.

Proof. Set $p:=\delta_{e}-\delta_{s_{1} s_{1}^{*}}$, and set $\mathscr{A}:=\left\{p \widehat{\star} f \widehat{\star} p \mid f \in \ell_{1}\left(S_{2}^{\mathbf{m}}\right)\right\}$. Proposition 1.15 and its proof (including the modified version of the proof of Proposition 1.8) show that $\mathscr{A}$ is a $*$-semisimple, unital, *-properly infinite Banach $*$-algebra in standard form.

The restriction of a bounded trace to a subalgebra is itself a bounded trace, so to prove that there is a non-zero, bounded trace on $\mathscr{A}$, it suffices to find a bounded trace on $\ell_{1}\left(S_{2}^{\mathbf{a}}\right)$ taking a non-zero value at an element in $\mathscr{A}$. Let $\tau$ in $\ell_{\infty}\left(S_{2}\right)$ be defined as in Example 3.8. Then $\tau \mid S_{2}^{\boldsymbol{\bullet}}$ is a bounded trace on $\ell_{1}\left(S_{2}^{\mathbf{a}}\right)$, and

$$
\left\langle p \widehat{\star} \delta_{s_{2} s_{2}^{*}} \widehat{\star} p, \tau \mid S_{2}^{\mathbf{\bullet}}\right\rangle=\tau\left(s_{2} s_{2}^{*}\right)=1 \neq 0,
$$

as desired.

3.18. Proposition. The Banach *-algebra $\ell_{1}\left(S_{2}^{\square}\right)$ is not hermitian.

Proof. Set $f:=\delta_{s_{1}}+\delta_{s_{2}}$ in $\ell_{1}\left(S_{2}^{\mathbf{\square}}\right)$. Then $f^{*} \widehat{\star} f=2 \delta_{e}$, and so

$$
\sigma\left(f^{*} \widehat{\star} f\right)=\{2\} \quad \text { and } \quad \nu\left(f^{*} \widehat{\star} f\right)=2 .
$$

On the other hand, $\left\|f^{n}\right\|_{1}=2^{n}$ for each $n$ in $\mathbb{N}$ because no cancellation occurs among the $2^{n}$ terms of the expression

$$
f^{n}=\sum_{i_{1}, \ldots, i_{n}=1}^{2} \delta_{s_{i_{1}} \ldots s_{i_{n}}} .
$$

This implies that $\nu(f)=2$ by the spectral radius formula (e.g., see [7, Theorem 2.3.8(iii)]).

In conclusion, we have

$$
\nu\left(f^{*} \widehat{\star} f\right)=2<4=\nu(f)^{2},
$$

and so $\ell_{1}\left(S_{2}^{\mathbf{\square}}\right)$ is not hermitian by [7, Proposition 3.1.8]. 
3.19. Remark. In fact, the proof of [7, Proposition 3.1.8] provides an explicit example of a self-adjoint element $k$ in $\ell_{1}\left(S_{2}^{\boldsymbol{\Xi}}\right)$ whose spectrum is not contained in $\mathbb{R}$.

To see this, we first show that the spectrum of the element $f:=\delta_{s_{1}}+\delta_{s_{2}}$ in $\ell_{1}\left(S_{2}^{\mathbf{\square}}\right)$ is given by

$$
\sigma(f)=\{\zeta \in \mathbb{C}|| \zeta \mid \leq 2\}
$$

The inclusion $\subseteq$ is clear because $\|f\|_{1}=2$.

Conversely, suppose that $\zeta$ belongs to $\mathbb{C} \backslash \sigma(f)$, and take $g=\sum_{s \in S_{2}} \alpha_{s} \delta_{s}$ in $\ell_{1}\left(S_{2}^{\mathbf{\square}}\right)$ such that $\left(f-\zeta \delta_{e}\right) \widehat{\star} g=\delta_{e}$, that is,

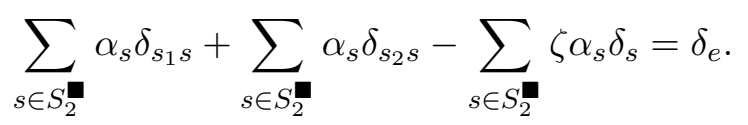

Identifying coefficients gives

$$
-\zeta \alpha_{e}=1, \quad \zeta \alpha_{s_{\mathbf{i}}^{*}}=0 \quad(\mathbf{i} \in \mathbf{I} \backslash\{\emptyset\}), \quad \alpha_{s}=\zeta \alpha_{s_{i} s} \quad\left(s \in S_{2}^{\mathbf{\bullet}}, i=1,2\right) .
$$

It follows that $\zeta \neq 0, \alpha_{e}=-1 / \zeta$, and, inductively,

$$
\begin{aligned}
\alpha_{s_{i_{1}} \ldots s_{i_{n}}} & =-\frac{1}{\zeta^{n+1}} & & \left(n \in \mathbb{N}, i_{1}, \ldots, i_{n} \in\{1,2\}\right), \\
\alpha_{s_{\mathbf{i}} s_{\mathbf{j}}^{*}} & =0 & & (\mathbf{i} \in \mathbf{I}, \mathbf{j} \in \mathbf{I} \backslash\{\emptyset\}) .
\end{aligned}
$$

Thus we have

$$
\infty>\|g\|_{1}=\frac{1}{|\zeta|}+\sum_{n=1}^{\infty}\left(\sum_{i_{1}, \ldots, i_{n}=1}^{2} \frac{1}{|\zeta|^{n+1}}\right)=\sum_{n=0}^{\infty} \frac{2^{n}}{|\zeta|^{n+1}}
$$

from which we conclude that $|\zeta|>2$. This completes the proof of (3.9).

Set $k:=\mathrm{i}\left(f^{*}-f\right)$ in $\ell_{1}\left(S_{2}^{\mathbf{*}}\right)$. Then $k$ is self-adjoint and satisfies

$$
\left(f^{*}+2 \delta_{e}\right) \widehat{\star}\left(f-2 \delta_{e}\right)=2 \mathrm{i}\left(k+\mathrm{i} \delta_{e}\right) .
$$

Assume towards a contradiction that $\sigma(k)$ is contained in $\mathbb{R}$. Then $k+\mathrm{i} \delta_{e}$ is invertible, and so $f-2 \delta_{e}$ is left invertible. By [7, Theorem 2.3.21(ii)], this implies that 2 is not in $\partial \sigma(f)$, contradicting (3.9). Hence, we conclude that the spectrum of $k$ is not contained in the real line; specifically, $-\mathrm{i}$ belongs to $\sigma(k)$.

We shall now show that the corner of $\ell_{1}\left(S_{2}^{\mathbf{n}}\right)$ considered in Theorem 3.17 also fails to be hermitian. By Proposition 1.15(iii), this result improves Proposition 3.18. The strategy of the proof is exactly the same as before, but the details are somewhat more complicated.

3.20. Proposition. The corner of $\ell_{1}\left(S_{2}^{\square}\right)$ corresponding to the projection $\delta_{e}-\delta_{s_{1} s_{1}^{*}}$ is not hermitian.

Proof. Set $p:=\delta_{e}-\delta_{s_{1} s_{1}^{*}}$, and set $\mathscr{A}:=\left\{p \widehat{\star} f \widehat{\star} p \mid f \in \ell_{1}\left(S_{2}^{\mathbf{\bullet}}\right)\right\}$. By Lemma 1.9, the spectral radius of an element $a$ in $\mathscr{A}$ is independent of 
whether it is calculated in $\ell_{1}\left(S_{2}^{\mathbf{a}}\right)$ or in $\mathscr{A}$, and so we shall denote it just by $\nu(a)$ (without any subscript).

Set

$$
g:=\delta_{s_{2}} \widehat{\star} p+\delta_{s_{2} s_{1} s_{2}^{*}}+p-\delta_{s_{2} s_{2}^{*}} \in \mathscr{A} .
$$

Then $g^{*} \widehat{\star} g=2 p$, and so $\nu\left(g^{*} \widehat{\star} g\right)=2$. On the other hand, an inductive argument shows that, for each integer $n \geq 2$, the $n$th power of $g$ is given by

$$
\begin{aligned}
g^{n}= & \delta_{s_{2}} \widehat{\star}\left(\delta_{s_{1}}+\delta_{s_{2}}\right)^{n-1} \widehat{\star}\left(p+\delta_{s_{1} s_{2}^{*}}\right) \\
& +\left(\delta_{e}+\delta_{s_{2}} \widehat{\star}\left(\sum_{m=0}^{n-2}\left(\delta_{s_{1}}+\delta_{s_{2}}\right)^{m}\right)\right) \widehat{\star}\left(p-\delta_{s_{2} s_{2}^{*}}\right) .
\end{aligned}
$$

By Lemma 3.7(ii), the terms of this expression have pairwise disjoint supports. This implies that $\left\|g^{n}\right\|_{1}=3 \cdot 2^{n}$, and so $\nu(g)=2$. In conclusion, we have

$$
\nu\left(g^{*} \widehat{\star} g\right)=2<4=\nu(g)^{2},
$$

and the result follows from [7, Proposition 3.1.8].

We conclude this section with another example of a standard property of $C^{*}$-algebras that fails for $\ell_{1}\left(S_{2}^{\mathbf{a}}\right)$.

3.21. Definition. A Banach algebra $\mathscr{A}$ is Arens regular if, for each pair $\left(\left(a_{m}\right)_{m=1}^{\infty},\left(b_{n}\right)_{n=1}^{\infty}\right)$ of bounded sequences in $\mathscr{A}$ and each bounded linear functional $\lambda: \mathscr{A} \rightarrow \mathbb{C}$, we have

$$
\lim _{m \rightarrow \infty}\left(\lim _{n \rightarrow \infty}\left\langle a_{m} b_{n}, \lambda\right\rangle\right)=\lim _{n \rightarrow \infty}\left(\lim _{m \rightarrow \infty}\left\langle a_{m} b_{n}, \lambda\right\rangle\right)
$$

whenever both the iterated limits exist.

A number of equivalent formulations of the notion of Arens regularity are given in [7, Theorem 2.6.17]. It is well known that every $C^{*}$-algebra is Arens regular (e.g., see [7, Corollary 3.2.37]). Using a technique introduced by Young in [21], we shall now show that this is not the case for $\ell_{1}\left(S_{2}^{\mathbf{m}}\right)$.

3.22. Proposition. The Banach *-algebra $\ell_{1}\left(S_{2}^{\mathbf{q}}\right)$ is not Arens regular.

Proof. Define $\lambda$ in $\ell_{\infty}\left(S_{2}\right)$ by

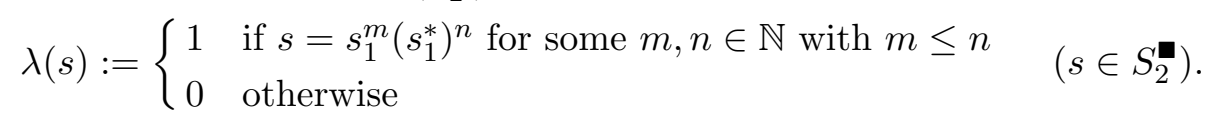

The sequences $\left(\delta_{s_{1}^{m}}\right)_{m=1}^{\infty}$ and $\left(\delta_{\left(s_{1}^{*}\right)^{n}}\right)_{n=1}^{\infty}$ in $\ell_{1}\left(S_{2}^{\mathbf{*}}\right)$ are bounded and satisfy

$$
\left\langle\delta_{s_{1}^{m}} \widehat{\star} \delta_{\left(s_{1}^{*}\right)^{n}}, \lambda\right\rangle=\lambda\left(s_{1}^{m}\left(s_{1}^{*}\right)^{n}\right)= \begin{cases}1 & \text { for } m \leq n, \\ 0 & \text { for } m>n .\end{cases}
$$

It follows that

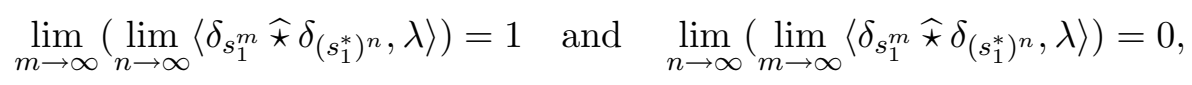

and so $\ell_{1}\left(S_{2}^{\mathbf{a}}\right)$ is not Arens regular. 
4. A unital *-algebra which is *-finite and properly infinite. A unital, finite $*$-algebra is automatically $*$-finite, and a unital, $*$-properly infinite $*$-algebra is automatically properly infinite. In this section we shall show that neither of these implications can be reversed, by constructing a unital $*$-algebra which is $*$-finite and properly infinite.

4.1. Algebraic semigroup algebras. For a semigroup $S$, we denote by $c_{00}(S)$ the set of finitely supported functions from $S$ to $\mathbb{C}$. This is a dense subalgebra of $\ell_{1}(S)$, termed the algebraic semigroup algebra of $S$. Clearly, $c_{00}(S)=\ell_{1}(S)$ if and only if $S$ has finite cardinality. In the case where $S$ has a zero element, $c_{00}\left(S^{\mathbf{\bullet}}\right)$ is a dense subalgebra of $\ell_{1}\left(S^{\mathbf{\square}}\right)$.

Suppose that $S$ has an involution. Then $c_{00}(S)$ is closed under the involution (3.4), and is thus a dense sub- $*$-algebra of $\ell_{1}(S)$. Similarly, in the case where $S$ has a zero element, $c_{00}\left(S^{\square}\right)$ is a dense sub-*-algebra of $\ell_{1}\left(S^{\square}\right)$.

4.2. Construction. Let $S$ be a semigroup with a neutral element $e$ and a zero element $\theta$. The free product of two copies of $S$ with amalgamation of the neutral elements and of the zero elements is given by

$$
\begin{aligned}
S * S:= & \left\{e^{\prime}, \theta^{\prime}\right\} \\
& \cup\left\{\left(w_{1}, i_{1}\right) \cdots\left(w_{n}, i_{n}\right) \mid n \in \mathbb{N}, w_{1}, \ldots, w_{n} \in S \backslash\{e, \theta\},\right. \text { and } \\
& \left.i_{1}, \ldots, i_{n} \in\{1,2\} \text { with } i_{j} \neq i_{j+1} \text { for each } j \in\{1, \ldots, n-1\}\right\} .
\end{aligned}
$$

We turn $S * S$ into a semigroup with neutral element $e^{\prime}$ and zero element $\theta^{\prime}$ by defining an operation $\circ$ as follows:

$$
\theta^{\prime} \circ w=\theta^{\prime}=w \circ \theta^{\prime}, \quad e^{\prime} \circ w=w=w \circ e^{\prime} \quad(w \in S * S),
$$

and, recursively, for $v=\left(v_{1}, i_{1}\right) \cdots\left(v_{m}, i_{m}\right)$ and $w=\left(w_{1}, j_{1}\right) \cdots\left(w_{n}, j_{n}\right)$ in $S * S \backslash\left\{e^{\prime}, \theta^{\prime}\right\}$

$$
v \circ w:=\left\{\begin{array}{rr}
\theta^{\prime} & \text { for } i_{m}=j_{1} \text { and } v_{m} w_{1}=\theta ; \\
e^{\prime} & \text { for } i_{m}=j_{1}, v_{m} w_{1}=e, \text { and } m=n=1 ; \\
\left(v_{1}, i_{1}\right) \cdots\left(v_{m-1}, i_{m-1}\right) \circ\left(w_{2}, j_{2}\right) \cdots\left(w_{n}, j_{n}\right) & \text { for } i_{m}=j_{1}, v_{m} w_{1}=e,
\end{array}\right.
$$

(Note: in the third and fourth cases, $\left(v_{1}, i_{1}\right) \cdots\left(v_{m-1}, i_{m-1}\right)$ should be ignored if $m=1$, and $\left(w_{2}, j_{2}\right) \cdots\left(w_{n}, j_{n}\right)$ should be ignored if $n=1$.)

Suppose that $S$ has an involution. Then we define an involution on $S * S$ by $\left(e^{\prime}\right)^{*}:=e^{\prime},\left(\theta^{\prime}\right)^{*}:=\theta^{\prime}$, and

$$
\left(\left(w_{1}, i_{1}\right) \cdots\left(w_{n}, i_{n}\right)\right)^{*}:=\left(w_{n}^{*}, i_{n}^{*}\right)\left(w_{n-1}^{*}, i_{n-1}^{*}\right) \cdots\left(w_{1}^{*}, i_{1}^{*}\right)
$$


for each $\left(w_{1}, i_{1}\right) \cdots\left(w_{n}, i_{n}\right)$ in $(S * S) \backslash\left\{e^{\prime}, \theta^{\prime}\right\}$, where

$$
i^{*}:=\left\{\begin{array}{ll}
2 & \text { for } i=1 \\
1 & \text { for } i=2
\end{array} \quad(i=1,2) .\right.
$$

The length of an element $w$ in $S * S$ is given by

$$
\operatorname{len}(w):= \begin{cases}-\infty & \text { for } w=\theta^{\prime} \\ 0 & \text { for } w=e^{\prime} \\ n & \text { for } w=\left(w_{1}, i_{1}\right) \cdots\left(w_{n}, i_{n}\right) \in(S * S) \backslash\left\{e^{\prime}, \theta^{\prime}\right\} .\end{cases}
$$

Clearly, for each $v$ and $w$ in $S * S$, we have

$$
\left\{\begin{aligned}
\operatorname{len}(v \circ w) & \leq \operatorname{len}(v)+\operatorname{len}(w) \\
\operatorname{len}\left(w^{*}\right) & =\operatorname{len}(w) \\
\operatorname{len}\left(w^{*} \circ w\right) & =2 \operatorname{len}(w)
\end{aligned}\right.
$$

As is customary, we shall henceforth omit the symbol $\circ$ and denote the semigroup operation in $S * S$ simply by juxtaposition of elements. Moreover, we shall drop the primes from $e^{\prime}$ and $\theta^{\prime}$ and write $e$ for the neutral element and $\theta$ for the zero element in $S * S$ (as well as in $\mathrm{S}$ ).

4.3. LEMmA. The only projections in $c_{00}\left(\left(S_{2} * S_{2}\right)\right.$ ) are 0 and $\delta_{e}$.

Proof. For $f$ in $c_{00}\left(\left(S_{2} * S_{2}\right)\right) \backslash\left\{0, \delta_{e}\right\}$, take $w=\left(w_{1}, i_{1}\right) \cdots\left(w_{n}, i_{n}\right)$ in $\left(S_{2} * S_{2}\right) \backslash\{e\}$ of maximal length such that $f(w) \neq 0$. Then $f\left(w^{*} w\right)=0$ because len $\left(w^{*} w\right)=2 n>n$.

On the other hand, by definition, we have

$$
\left(f^{*} \star f\right)\left(w^{*} w\right)=\sum_{\substack{u v=w^{*} w \\ u, v \in S_{2} * S_{2}}} \overline{f\left(u^{*}\right)} f(v) .
$$

Suppose that $u$ and $v$ in $S_{2} * S_{2}$ satisfy $u v=w^{*} w$. Then $\operatorname{len}(u)+\operatorname{len}(v) \geq 2 n$ by (4.1). Since $f(t)=0$ for each $t$ in $\left(S_{2} * S_{2}\right)$ with $\operatorname{len}(t)>n$, the only non-zero contributions to the sum on the right-hand side of (4.2) arise for $\operatorname{len}(u)=\operatorname{len}(v)=n$. But the only $u$ and $v$ of length $n$ in $S_{2} * S_{2}$ with $u v=w^{*} w$ are $u=w^{*}$ and $v=w$. It follows that

$$
\left(f^{*} \widehat{\star} f\right)\left(w^{*} w\right)=\overline{f\left(\left(w^{*}\right)^{*}\right)} f(w)=|f(w)|^{2} \neq 0 .
$$

In conclusion, we have $f \neq f^{*} \widehat{\star} f$, and so $f$ is not a projection.

4.4. Proposition. The unital *-algebra $c_{00}\left(\left(S_{2} * S_{2}\right)\right)$ is *-finite and properly infinite.

Proof. Lemma 4.3 shows that $c_{00}\left(\left(S_{2} * S_{2}\right)\right)$ is $*$-finite.

On the other hand, set $a_{i}:=\delta_{\left(s_{i}, 1\right)}$ and $b_{i}:=\delta_{\left(s_{i}^{*}, 1\right)}$ in $c_{00}\left(\left(S_{2} * S_{2}\right)\right.$ ) for $i=1,2$. Then (1.1) is satisfied, and so we conclude that $c_{00}\left(\left(S_{2} * S_{2}\right)\right.$ 叫 is properly infinite. 
4.5. Remark. The unital Banach *-algebra $\ell_{1}\left(\left(S_{2} * S_{2}\right)\right)$ is properly infinite because it contains $c_{00}\left(\left(S_{2} * S_{2}\right)\right.$, which is properly infinite, as a unital subalgebra. We have not been able to determine whether or not $\ell_{1}\left(\left(S_{2} * S_{2}\right)\right)$ is $*$-properly infinite. If $\ell_{1}\left(\left(S_{2} * S_{2}\right)\right)$ fails to be $*$-properly infinite, then it would be of interest to determine its traces (cf. Question 1.11(iv)).

Acknowledgements. We are grateful to John Duncan (Arkansas) for sharing with us his insights into semigroup Banach algebras and for drawing our attention to reference [19], and to Mikael Rørdam (Odense) for his advice on properly infinite $C^{*}$-algebras.

\section{References}

[1] B. A. Barnes, Representations of the $\ell_{1}$-algebra of an inverse semigroup, Trans. Amer. Math. Soc. 218 (1976), 361-396.

[2] S. Bowling and J. Duncan, First order cohomology of Banach semigroup algebras, Semigroup Forum 56 (1998), 130-145.

[3] A. H. Clifford and G. B. Preston, The Algebraic Theory of Semigroups, Vol. I, Math. Surveys, 7, Amer. Math. Soc., Providence, 1961.

[4] J. Cuntz, Simple $C^{*}$-algebras generated by isometries, Comm. Math. Phys. 57 (1977), 173-185.

[5] -, Murray-von Neumann equivalence of projections in infinite simple $C^{*}$-algebras, Rev. Roumaine Math. Pures Appl. 23 (1978), 1011-1014.

[6] -, K-theory for certain $C^{*}$-algebras, Ann. of Math. 113 (1981), 181-197.

[7] H. G. Dales, Banach Algebras and Automatic Continuity, London Math. Soc. Monograph 24, Clarendon Press, Oxford, 2000.

[8] J. Duncan and J. H. Williamson, Spectra of elements in the measure algebra of a free group, Proc. Roy. Irish Acad. Sect. A 82 (1982), 109-120.

[9] T. Fack, Finite sums of commutators in $C^{*}$-algebras, Ann. Inst. Fourier (Grenoble) 32 (1982), 129-137.

[10] B. E. Johnson, Symmetric amenability and the nonexistence of Lie and Jordan derivations, Math. Proc. Cambridge Philos. Soc. 120 (1996), 455-473.

[11] R. V. Kadison and J. R. Ringrose, Fundamentals of the Theory of Operator Algebras, Vols. I-II, Academic Press, San Diego, 1983-1986.

[12] N. J. Laustsen, Commutators of operators on Banach spaces, J. Operator Theory, to appear.

[13] -, On ring-theoretic (in)finiteness of Banach algebras of operators on Banach spaces, Glasgow J. Math., to appear.

[14] M. Mathieu and A. R. Villena, The structure of Lie derivations on $C^{*}$-algebras, preprint.

[15] T. W. Palmer, Banach Algebras and the General Theory of ${ }^{*}$-algebras, Vol. II, Cambridge Univ. Press, 2001.

[16] A. L. T. Paterson, Groupoids, Inverse Semigroups, and Their Operator Algebras, Progress Math. 170, Birkhäuser, Boston, 1999.

[17] C. Pop, Finite sums of commutators, Proc. Amer. Math. Soc. 130 (2002), 3039-3041.

[18] J. N. Renault, A Groupoid Approach to $C^{*}$-algebras, Lecture Notes in Math. 793, Springer, New York, 1980. 
[19] J. R. Wordingham, Topics in Semigroup Algebras, PhD thesis, Univ. of Stirling, 1982.

[20] - The left regular *-representation of an inverse semigroup, Proc. Amer. Math. Soc. 86 (1982), 55-58.

[21] N. J. Young, The irregularity of multiplication in group algebras, Quart. J. Math. Oxford, Series (2) 24 (1973), 59-62.

Department of Pure Mathematics

University of Leeds

Leeds LS2 9JT, England

E-mail: pmt6hgd@amsta.leeds.ac.uk read@amsta.leeds.ac.uk
Department of Mathematics University of Copenhagen Universitetsparken 5 DK-2100 København Ø, Denmark

E-mail: laustsen@math.ku.dk

Received February 22, 2002

Revised version September 5, 2002 